\title{
Effects of a high-intensity interval training program versus a moderate-intensity continuous training program on maximal oxygen uptake and blood pressure in healthy adults: study protocol for a randomized controlled trial
}

Víctor Hugo Arboleda Serna ${ }^{1}$, Elkin Fernando Arango Vélez ${ }^{1}$, Rubén Darío Gómez Arias $^{2}$ and Yuri Feito ${ }^{3 *}$ (D)

\begin{abstract}
Background: Participation in aerobic exercise generates increased cardiorespiratory fitness, which results in a protective factor for cardiovascular disease and all-cause mortality. High-intensity interval training might cause higher increases in cardiorespiratory fitness in comparison with moderate-intensity continuous training; nevertheless, current evidence is not conclusive. To our knowledge, this is the first study to test the effect of high-intensity interval training with total load duration of 7.5 min per session.

Methods: A randomized controlled trial will be performed on two groups of healthy, sedentary male volunteers $(n=44)$. The study protocol will include 24 exercise sessions, three times a week, including aerobic training on a treadmill and strength training exercises. The intervention group will perform 15 bouts of $30 \mathrm{~s}$, each at an intensity between $90 \%$ and $95 \%$ of maximal heart rate. The control group will complete 40 min of continuous exercise, ranging between $65 \%$ and $75 \%$ of maximal heart rate. The primary outcome measure to be evaluated will be maximal oxygen uptake $\left(\mathrm{VO}_{2 \mathrm{max}}\right)$, and systolic and diastolic blood pressure will be evaluated as secondary outcome measures. Waist circumference, body mass index, and body composition will also be evaluated.

Discussion: Epidemiological evidence shows the link between $\mathrm{VO}_{2 \max }$ and its association with chronic conditions that trigger CVD. Therefore, finding ways to improve $\mathrm{VO}_{2 \max }$ and reduce blood pressure it is of vital importance to public health.
\end{abstract}

Trial registration: NCT02288403. Registered on 4 November 2014.

Keywords: Blood pressure, Exercise, High-intensity interval training, Randomized controlled trial, $\mathrm{VO}_{2 \max }$

\footnotetext{
* Correspondence: yfeito@kennesaw.edu

${ }^{3}$ Department of Exercise Science \& Sport Management, Kennesaw State

University, 520 Parliament Garden Way, NW, MD 4104 Kennesaw, GA, USA

Full list of author information is available at the end of the article
} 


\section{Background}

Maximal oxygen consumption $\left(\mathrm{VO}_{2 \max }\right)$ provides a measure of the maximal volume of oxygen that the body consumes via the respiratory system and is transported through the bloodstream to be used to release energy in the cell. $\mathrm{VO}_{2 \max }$ is currently deemed the best indicator to assess cardiorespiratory fitness $[1,2]$ and is considered one of the most important predictors of cardiovascular disease in both men and women, even when taking into consideration smoking history, hypertension, and dyslipidemias. In addition, $\mathrm{VO}_{2 \max }$ is directly related to cardiovascular health, and its improvement has been linked to decreases in risk of death from cardiovascular disease [3]. For every metabolic equivalent gained in $\mathrm{VO}_{2 \text { max }}$, mortality rates have been shown to decrease by about 8-17 \% [3-5] (one metabolic equivalent $=3.5 \mathrm{ml} /$ ( $\mathrm{kg}$ min)). Laukkanen et al. [6] studied the association between low levels of $\mathrm{VO}_{2 \max }(<27.6 \mathrm{ml} /(\mathrm{kg} \mathrm{min}))$ and premature death and found that unfit men had more than twice the risk of overall death (relative risk, 2.76; 95 $\%$ confidence interval, 1.43-5.33) and over three times the risk of cardiovascular-disease-related death (relative risk $=3.09$; $95 \%$ confidence interval, 1.10-9.56) in comparison with fit men $\left(\mathrm{VO}_{2 \max }>37.1 \mathrm{ml} / \mathrm{kg} / \mathrm{min}\right)$.

Moderate-intensity continuous training methods and high-intensity interval training are typically prescribed to increase $\mathrm{VO}_{2 \max }$. The latter has been generally used with athletes; however, this modality has recently been used on untrained individuals with cardiometabolic disorders and cardiovascular disease [7]. Considering the inverse relationship between intensity and duration of exercise, it is important to consider the total volume of the exercise session when prescribing this type of program. High-intensity interval training protocols are typically classified utilizing short intervals (around $30 \mathrm{~s}$ ) and intensities near maximum (80-100 \% peak heart rate), whereas moderate-intensity continuous training programs typically remain between $60 \%$ and $75 \%$ of peak heart rate). In addition, an alternative term has been suggested [7] to denote training programs that utilize intensities over $100 \%$ peak heart rate (sprint interval training). In this study, we will employ a high-intensity interval training protocol of low volume.

The worldwide survey on fitness trends has ranked high-intensity interval training at the top of the top 20 trends for the past two consecutive years [8, 9], which supports use in the general populations, and reveals interest in this training methodology. However, although the benefits of these two modalities are a matter of discussion, comparisons between the two training modalities suggest that high-intensity interval training can produce faster and more significant adaptations in $\mathrm{VO}_{2 \max }$ [10-16]. The results of several meta-analyses have shown slightly higher improvements in $\mathrm{VO}_{2 \max }$ with high-intensity interval training than moderateintensity continuous training; however, many of these studies used short interventions with few weekly training sessions $[17,18]$. In addition, it is important to point out that in a recent meta-analysis on the effects of interval training on $\mathrm{VO}_{2 \max }$, only 4 of the 21 studies that met the inclusion criteria were randomized controlled trials [19]. Milanović et al. [20] pointed out the large improvements in $\mathrm{VO}_{2 \max }$ when comparing high-intensity interval training with control subjects who do not exercise (5.5 $\mathrm{ml} /(\mathrm{kg} \mathrm{min}))$; meanwhile, when comparing highintensity interval training with aerobic training, this improvement was reduced $(1.2 \mathrm{ml} /(\mathrm{kg} \mathrm{min}))$.

High blood pressure is one of the most common health disorders; it is associated with an increase in the incidence of all-cause mortality and cardiovascular disease. It has been reported that people with systolic blood pressure between 130 and $139 \mathrm{mmHg}$, and diastolic blood pressure between 85 and $89 \mathrm{mmHg}$ have a greater risk of cardiovascular events than those who present with optimal blood pressure values (systolic blood pressure $<120 \mathrm{mmHg}$, diastolic blood pressure $<80 \mathrm{mmHg}$ ), with hazard ratio $=2.5$; $95 \%$ confidence interval $=1.6-$ 4.1 for women, and hazard ratio $=1.6$; $95 \%$ confidence interval $=1.1-2.2$ for men. Likewise, individuals with high blood pressure (systolic blood pressure $>140$ $\mathrm{mmHg}$, diastolic blood pressure $>90 \mathrm{mmHg}$, or those who use antihypertensive medication) have greater risk of cardiovascular events than individuals with optimal values [21]. Moreover, researchers have found an inverse association between cardiorespiratory fitness and the risk of high blood pressure, where for every $1 \mathrm{ml} /(\mathrm{kg} \mathrm{min})$ increase in $\mathrm{VO}_{2 \max }$, the prevalence of hypertension is reduced by 1.04 times (95\% confidence interval, 1.05-1.02) [22]. In their recent meta-analysis looking at the effects of exercise training in blood pressure, Cornelissen and Smart [23] reported decreases in systolic blood pressure and diastolic blood pressure of $3.5 \mathrm{mmHg}$ (95\% confidence interval, 2.3-4.6), and $2.5 \mathrm{mmHg}$ (95\% confidence interval, 1.7-3.2), respectively, with aerobic exercise. Studies comparing continuous training, high-intensity interval training and control subjects who did not exercise and were hypertensive reported significant mean decreases in systolic blood pressure of $8 \mathrm{mmHg}$ in all groups [12], and mean decreases in systolic blood pressure and diastolic blood pressure with high-intensity interval training of 12 and $8 \mathrm{mmHg}$, respectively, in comparison with continuous training subjects, who obtained non-significant decreases of 4.5 and $3.5 \mathrm{mmHg}$ [24].

Even though exercising is a fundamental aspect in primary prevention, treatment, and control of hypertension, the optimal frequency, intensity, time, and mode of exercise to reduce systolic blood pressure and diastolic blood pressure values are still unclear [25]. In this sense, it is 
necessary to conduct randomized controlled trials that allow us to examine and confirm the cause-effect relationship between high-intensity interval training and $\mathrm{VO}_{2 \max }$, as well as between high-intensity interval training and blood pressure. Although evidence exists that suggests the benefits of high-intensity interval training in $\mathrm{VO}_{2 \max }$ and blood pressure in individuals with cardiovascular disease [7], the effect of these programs among relatively healthy young men with low levels of physical activity is unclear $[7,19,20]$. Current evidence regarding this topic is limited; thus, this project aims to provide clarity regarding the appropriate dose (frequency, volume, and intensity) to generate significant improvements in $\mathrm{VO}_{2 \max }$ and decreases in systolic and diastolic blood pressure in healthy individuals, as the potential benefits of this type of training are still unclear in this group [26, 27] Taking into account that lack of time is typically the most common cited barrier to engagement in exercise training [28], we believe that this study is novel in its approach, as, to our knowledge, we are the first to examine the effects of a low-volume high-intensity interval training protocol of only $7.5 \mathrm{~min}$ per session. Although other studies have utilized less work time per session than this study, those investigations utilized near maximal protocols $\left(\geq 100 \% \mathrm{VO}_{2 \max }\right)$ and were not randomized control trials [29, 30]. In addition, our comparison group will use a treadmill as a training modality, which is an activity typical for activities of daily living; thus, providing greater validity to our final results.

\section{Objectives}

The main objective of this randomized controlled trial is to determine the effect of a high-intensity interval training program in comparison with a moderate-intensity continuous training program on $\mathrm{VO}_{2 \max }$. A secondary objective is to examine the effects of both training programs on systolic and diastolic blood pressure.

\section{Hypothesis}

The primary hypothesis of this study is that compared with an 8 week moderate-intensity continuous training program, a high-intensity interval training program will have a significant effect on $\mathrm{VO}_{2 \max }$ in a group of 18-44year-old men. A secondary hypothesis proposes that, in comparison with a moderate-intensity continuous training program, there is a significant positive effect on systolic and diastolic blood pressure with a high-intensity interval training program.

\section{Methods}

\section{Study design}

This study is a randomized controlled trial with parallel arms conducted and reported in accordance with $\mathrm{CON}$ SORT guidelines for non-pharmacologic treatment [31].

\section{Recruiting}

Volunteers will be recruited through posters sent via email to the academic community throughout the Universidad de Antioquia, and located in various places on the university campus and nearby educational institutions, as well as via Facebook. Recruiting will finish once the number of volunteers required is complete. There will be no paid incentives to participate in this study.

\section{Research site}

Assessments will be performed at the Laboratory for Physical Activity and Sports Sciences, Universidad de Antioquia. The interventions will be conducted at the sporting facilities of the Universidad de Antioquia.

\section{Eligibility criteria}

Forty-four healthy but sedentary $(\leq 150 \mathrm{~min}$ of aerobic exercise a week (as reported using the Global Physical Activity Questionnaire [32]) young men between 18 and 44 years old, who are enrolled in the General Social Security Health System in Colombia [33] will be recruited for this study. Prior to starting the program, all participants must accept their voluntary participation by signing an informed consent form. The Research Ethics Committee at the Universidad de Antioquia School of Public Health in Medellín, Colombia approved the study protocol. Subjects will be excluded if they are currently enrolled in a high-intensity interval training program, smoked, have a history of cardiovascular disease, coronary disease, arrhythmias, cardiac insufficiency, hypertension (history of hypertension, currently taking pharmacological agents, or blood pressure $\geq 140 / 90 \mathrm{mmHg}$ ), diabetes mellitus, are taking anticoagulants, beta blockers, calcium antagonists, bronchodilators, or steroids, or have cognitive impairments or neuromuscular or musculoskeletal disorders that could affect their participation in the exercise program. A physician specializing in sports medicine will evaluate all participants, and will confirm their participation in accordance with the study criteria.

\section{Randomization}

The random allocation sequence will be created in permuted blocks of four and six using random allocation software [34]. Allocation concealment will be achieved using sequentially numbered, opaque sealed envelopes [35]. Volunteers will be assigned to an intervention or control group in a 1:1 ratio, depending on the order they enroll into the study (after evaluation of baseline outcomes). An independent researcher will conduct the process. Researchers in charge of recruiting, outcome evaluation, and analysis will be blinded in group assignment, and personnel in charge of evaluating and conducting interventions will be trained following the protocols designed for those objectives. 


\section{Adherence strategies}

After obtaining volunteers' consent, volunteers will be sent reminders to attend their sessions through text messages, emails, and phone calls. Volunteers may contact the intervention coordinator and researchers 24 hours a day via email or by calling their cell phones.

\section{Information management}

Files with participant's information will be coded with individual identification codes. Data will be stored in a locked filing cabinet and in a password-protected computer, to which only researchers will have access. The computer password will be changed once a month throughout the duration of the research study; furthermore, a backup copy of all the information will be made once a month.

\section{Interventions}

Twenty-four sessions of aerobic exercise on a treadmill, plus strength training exercises, will be completed as a non-differential co-intervention in both groups, based on the recommendations set forth by the American College of Sports Medicine for healthy beginners or intermediate-level adults [36]. Each participant will take part in three-weekly sessions of personalized training on alternate days at the same time of day for eight-weeks, supervised by a qualified trainer who is knowledgeable of the study protocol and procedures. Throughout each session, the intensity of the subject's effort will be monitored using a Polar ${ }^{\ominus}$ FT1 heart rate monitor, and the compliance with each protocol will also be recorded, in forms designed for this purpose. Participants will warm up during the first $5 \mathrm{~min}$ on a treadmill at $50-60 \%$ of maximal heart rate, and then follow the exercise protocol assigned to them (intervention or control), with a 3 min recovery period at 40-50\% of maximal heart rate. Finally, they will perform two sets of 8 to 12 repeats in a circuit training (non-differential co-intervention), consisting of eight strength training exercises mainly involving large muscle groups at $60-70 \%$ of a one-repetition maximum, which equals a six or seven in the OMNI resistance exercise scale [37]. The objective of this portion of the intervention is simply to introduce these participants to the benefits of resistance training twice per week, as suggested by the American College of Sports Medicine [36]. We believe this portion of the intervention will not have an impact on any of the primary outcomes of our study.

The intervention group (high-intensity interval training), will undergo 15 bouts of 30 s loads at $90-95 \%$ of maximal heart rate followed by a $60 \mathrm{~s}$ recovery at 50-55 $\%$ of baseline $\mathrm{VO}_{2 \max }$ with an effort:recovery ratio of 1:2. The intensity of each high-intensity interval training session will be reached and adjusted manually throughout the session by a trained investigator manipulating the speed of the treadmill to reach the target heart rate, while maintaining a constant $10 \%$ elevation. It is worth highlighting that this intervention proposes short load times with double recovery time, which is different from those used in similar studies, which provided long load times with a recovery time similar to or less than the load time [11, 14, 24, 38, 39]. Other studies used an intervention with fewer but longer intervals [12, 40], and one study used a shorter load time [11]. Nevertheless, the total load time per session in those previous studies was greater than the time proposed in this randomized controlled trial, which is $21.5 \mathrm{~min}$ total $(7.5 \mathrm{~min}$ at 90 $95 \%$ of maximal heart rate and $14 \mathrm{~min}$ of recovery). Furthermore, the intention of this (high-intensity interval training) intervention is to show whether a shorter total time of high-intensity loads per session can generate similar or higher increases in $\mathrm{VO}_{2 \max }$ than longer load interventions. This change may be important, considering the fact that current public health guidelines regarding the minimum dose of exercise required to improve cardiovascular health seem difficult to follow, owing to barriers related to users' lack of time [28].

The control group (moderate-intensity continuous training) will do $40 \mathrm{~min}$ of continuous treadmill exercise at $65-75 \%$ of maximal heart rate. Throughout the intervention, volunteers will be encouraged to continue with their regular daily routine and not engage in any other exercises different form the ones proposed in the research study. Furthermore, they will asked to wear an Omron $^{\bullet} \mathrm{HJ}-112$ pedometer [41] to monitor their ambulatory daily activity.

\section{Evaluation of outcomes}

The evaluation of primary and secondary outcome measures will follow the order established by the American College of Sports Medicine for health-related physical assessment [42] and will be conducted in the following order: blood pressure (systolic and diastolic) measurements, waist circumference, height, weight, body composition, and cardiovascular endurance test. The final evaluations will be conducted within 72 hours of the last training session. Prior to each evaluation, all participants will be asked to avoid alcohol and drink plenty of water 24 hours before the testing session. In addition, they will be asked to avoid caffeine 6 hours prior to the evaluation and to avoid any vigorous exercise. Participants will be encouraged to arrive well rested, having had 6-8 hours of sleep the night before, and to wear comfortable athletic clothing.

The evaluation of blood pressure will be conducted first during each testing session and will be performed using an Omron ${ }^{\oplus}$ M3 HEM-7200-E (Omron Healthcare, 
Co., Ltd., Kyoto, Japan) automatic blood pressure monitor [43] to measure both systolic and diastolic blood pressure measures. Participants will be asked to sit and remain seated for $5 \mathrm{~min}$ with their feet on the ground and their arms resting at the heart level [44]. Two blood pressure measurements will be performed on the left arm with a $1 \mathrm{~min}$ pause between measurements. The average of both values will be used as the final systolic and diastolic measurements.

To assess body composition, an Omron ${ }^{\circ}$ HBF-510 (Omron Healthcare, Inc. Bannockburn, IL, USA) bioelectrical impedance machine will be used, following manufacturer protocols. Briefly, individuals will be instructed to wear light clothing, and stand barefoot on the device, which includes four electrodes on the feet and two electrodes on the handle. Participants will each be asked to hold the handle in their hands during the measurement, with the arms extended in front of them at eye level. The Omron ${ }^{\circ}$ HBF-510 will measure weight, percentage of body fat, percentage of lean tissue and body mass index. To measure height, volunteers will be asked to stand on a stadiometer without shoes and with their heels together, while looking straight ahead. Participants will each be asked to inhale deeply, and hold their breath briefly while the evaluator slides the stadiometer set square to the top of their head and records the height in centimeters. Waist circumference will be measured following standard procedures, at the mid-point between the lower costal edge and the iliac crest [45].

Measurements of $\mathrm{VO}_{2 \max }$ will be obtained using a graded exercise test on a treadmill (Trackmaster ${ }^{\circ}$ model TMX 425C) using a portable $\mathrm{K}_{4} \mathrm{~b}^{2}$ gas analyzer (Cosmed Inc., IL, USA), which has been validated and utilized in previous studies [46-50]. The equipment will be warmed up and calibrated following standard procedures prior to each evaluation. Before every assessment and after each test, components will be cleaned and disinfected following the manufacturer's recommendations, to guarantee valid measurements and to ensure participants' safety.

To conduct the stress test, participants will warm up for $3 \mathrm{~min}$ at $5 \mathrm{~km} / \mathrm{h}$. Immediately following the warmup period, the test will begin at a speed of $4 \mathrm{~km} / \mathrm{h}$ and will increase by $1 \mathrm{~km} / \mathrm{h}$ every minute with a constant 10 $\%$ gradient $\left(5.7^{\circ}\right)$ until maximal effort is achieved. To determine $\mathrm{VO}_{2 \max }$, the following criteria will be followed: respiratory quotient $>1.10$, maximal heart rate $\geq 95 \%$, following the 220-age equation [44], and reach a $\mathrm{VO}_{2}$ plateau despite an increase in load $\left(\mathrm{VO}_{2}\right.$ does not increase or go less than $2 \mathrm{ml} /(\mathrm{kg} \mathrm{min}))[38,51]$. The test will end when the criterion is achieved or when the participant asks to stop the test because of fatigue or some other reason.

\section{Statistical considerations Power and sample size}

To calculate sample size, a $3.5 \mathrm{ml} /(\mathrm{kg} \mathrm{min})$ difference of measurements in $\mathrm{VO}_{2 \max }$ was considered as a minimum effect to decrease the risk of cardiovascular disease $[4,5]$ with standard deviations for the intervention group and the control group of 2.6 and 4.6, respectively [14], assuming a $95 \%$ confidence level, $5 \%$ alpha error, and 20 $\%$ beta error, with a 1:1 ratio between groups. Using Epidat software (version 4.0), it was found that a sample of 20 individuals per group would be needed, plus an estimated $10 \%$ for possible losses.

\section{Analysis plan}

All analyses will be conducted according to the intention-to-treat principle, using a normality test, homoscedasticity test, or linearity test as necessary, to verify assumptions for the use of parametric statistics. As long as all assumptions of normality are met, analysis of covariance (ANCOVA) [52] will be used to control for the baseline values and adjust for possible confounding variables. In this regard, means and standard deviations will be used as summary measures. In the event that the requirements for parametric analysis are not met, a Mann-Whitney $\mathrm{U}$ test will be adopted, and values will be reported in medians and interquartile ranges. A twotailed significance test with a $P<0.05$ and a $95 \%$ confidence level will be used. To manage missing data, multiple imputation procedures will be used to replace missing values for the primarily variables of interest, following the guidelines set forth by Stern et al. [53]. Briefly, multiple imputation procedures create several sets of plausible imputed data, based on the Bayesian approach, and combine the results obtained with each one to create a value for each missing data point. Stata software (version 13) will be used to carry out all statistical procedures.

\section{Discussion}

$\mathrm{VO}_{2 \max }$ is considered one of the most important predictors of CVD mortality; in addition to smoking, high blood pressure (hypertension) and cholesterol disorders, low CRF levels increase the risk of CVD, is associated with morbidity and mortality from the same cause, as well as with all-cause mortality [4-6, 54-58]. Epidemiological evidence shows the link between $\mathrm{VO}_{2 \max }$ and its association with chronic conditions that trigger CVD, and subsequently increase the likelihood of death [56]. Similarly, high blood pressure is associated with increased risk of CVD [21]. Therefore, finding ways to increase $\mathrm{VO}_{2 \max }$ and reduce SBP and DBP it is of vital importance to public health. Recently the cardiorespiratory improvements obtained with HIIT have been examined; however, the optimal dose necessary requires 
further inquiry [20]; thus, it is important to develop RCT's as the one proposed in this study, which will clarify and provide insight to the cause-effect relationship between HIIT and $\mathrm{VO}_{2 \max }$, as well as HIIT and SBPDBP. This research is novel since it is the first to examine a HIIT workout with a heavy load with a total length 7.5 minutes per session, compared with other studies using longer load periods [11, 13, 14, 38, 39]. This project is of utmost importance, as exercise adherence is typically hindered by the lack needed to complete a typically traditionally design exercise program [28]. Another strength of this study is the design used (RCT), which has the power to detect differences in the effect on $\mathrm{VO}_{2 \max }$ produced by a HIIT vs MICT program.

\section{Trial status}

The call for participants started on April 25, 2015. We have enrolled 29 volunteers as of the submission of this manuscript. Of those 29, 18 have finished the interventions, and two withdrew from the study for personal reasons. The remaining volunteers are active. Recruitment of all volunteers is expected to finish in June 2016.

\section{Abbreviations}

ANCOVA, analysis of covariance; CONSORT, Consolidated Standards of Reporting Trials; $\mathrm{VO}_{2 \text { max }}$ maximal oxygen uptake.

\section{Acknowledgements}

The authors thank the support received from Universidad de Antioquia Sustainability Strategy CODI 2013-2014

\section{Authors' contributions}

VHAS, EA, and YF conceived and designed the study. VHAS, EA, RDG, and YF developed the study design. VHAS and EA are responsible for the management of the study, recruitment, and evaluation of outcomes. VHAS, $E A$, and RG calculated the power and sample size, and developed the statistical analysis plan for outcomes. YF completed the translation of the final manuscript. All of the authors reviewed and approved the final manuscript.

\section{Authors' information}

VHAS is Associate Professor and EA is Professor at Instituto de Educación Física Universidad de Antioquia. RG is Professor at Facultad Nacional de Salud Pública Universidad de Antioquia. YF is Associate Professor at the Department of Exercise Science \& Sport Management, Kennesaw State University.

\section{Competing interests}

The authors declare no competing interests.

\section{Ethics approval and consent to participate}

The Research Ethics Committee at Universidad de Antioquia School of Public Health in Medellín, Colombia approved the study protocol.

\section{Author details}

${ }^{1}$ Grupo de Investigación en Actividad Física para la Salud (AFIS), Instituto de Educación Física, Universidad de Antioquia UdeA, Calle 70 No. 52-21, Medellín, Colombia. ${ }^{2}$ Facultad Nacional de Salud Pública, Universidad de Antioquia UdeA, Calle 70 No. 52-21, Medellín, Colombia. ${ }^{3}$ Department of Exercise Science \& Sport Management, Kennesaw State University, 520 Parliament Garden Way, NW, MD 4104 Kennesaw, GA, USA.

Received: 15 March 2016 Accepted: 22 July 2016

Published online: 18 August 2016

\section{References}

1. Adams KJ. Exercise physiology. In: Swain DP, editor. ACSM's resource manual for guidelines for exercise testing and prescription. Philadelphia, PA: Lippincott Williams \& Wilkins; 2014. p. 57-8.

2. Kenney WL, Wilmore JH, Costill DL. Adaptations to aerobic and anaerobic training. In: Physiology of sport and exercise. Champaign, IL: Human Kinetics; 2015. p. 261-90.

3. Blair SN, et al. Influences of cardiorespiratory fitness and other precursors on cardiovascular disease and all-cause mortality in men and women. JAMA. 1996;276(3):205-10.

4. Gulati M, et al. Exercise capacity and the risk of death in women: the St James Women Take Heart Project. Circulation. 2003;108(13):1554-9.

5. Myers J, et al. Exercise capacity and mortality among men referred for exercise testing. N Engl J Med. 2002;346(11):793-801.

6. Laukkanen JA, et al. Cardiovascular fitness as a predictor of mortality in men. Arch Intern Med. 2001;161(6):825-31.

7. Weston KS, Wisløff U, Coombes JS. High-intensity interval training in patients with lifestyle-induced cardiometabolic disease: a systematic review and meta-analysis. Br J Sports Med. 2014;48(16):1227-34.

8. Thompson WR. Worldwide survey of fitness trends for 2014. ACSM's Health Fit J. 2013;17(6):10-20.

9. Thompson WR. Worldwide survey of fitness trends for 2015. ACSM's Health Fit J. 2014;18(6):8-17.

10. Gibala MJ, McGee SL. Metabolic adaptations to short-term high-intensity interval training: a little pain for a lot of gain? Exerc Sport Sci Rev. 2008; 36(2):58-63.

11. Helgerud J, Høydal K, Wang E, Karlsen T, Berg P, Bjerkaas M, et al. Aerobic high-intensity intervals improve $\mathrm{VO}_{2 \text { max }}$ more than moderate training. Med Sci Sports Exerc. 2007; 39(4):665.

12. Nybo $L$, et al. High-intensity training versus traditional exercise interventions for promoting health. Med Sci Sports Exerc. 2010;42(10):1951-8.

13. Tjønna $A E$, et al. Low-and high-volume of intensive endurance training significantly improves maximal oxygen uptake after 10-weeks of training in healthy men. PLoS One. 2013;8(5):e65382.

14. Tsekouras YE, Magkos F, Kellas Y, Basioukas KN, Kavouras SA, Sidossis LS. High-intensity interval aerobic training reduces hepatic very low-density lipoprotein-triglyceride secretion rate in men. Am J Physiol Endocrinol Metab. 2008:295(4):E851-8

15. Wisløff $U$, et al. Superior cardiovascular effect of aerobic interval training versus moderate continuous training in heart failure patients a randomized study. Circulation. 2007;115(24):3086-94.

16. Ziemann E, et al. Aerobic and anaerobic changes with high-intensity interval training in active college-aged men. J Strength Cond Res. 2011; 25(4):1104-12.

17. Bacon AP, Carter RE, Ogle EA, Joyner $M J . V_{2}$ max trainability and high intensity interval training in humans: a meta-analysis. PLoS One. 2013;8(9): e73182.

18. Hwang $C-L$, Wu $Y-T$, Chou $C-H$. Effect of aerobic interval training on exercise capacity and metabolic risk factors in people with cardiometabolic disorders: a meta-analysis. J Cardiopulm Rehabil Prev. 2011;31(6):378-85.

19. Sloth M, Sloth D, Overgaard K, Dalgas U. Effects of sprint interval training on $\mathrm{VO}_{2 \max }$ and aerobic exercise performance: a systematic review and metaanalysis. Scand J Med Sci Sports. 2013;23(6):e341-52.

20. Milanović Z, Sporiš G, Weston M. Effectiveness of high-intensity interval training $(\mathrm{HIT})$ and continuous endurance training for $\mathrm{VO}_{2 \max }$ improvements: a systematic review and meta-analysis of controlled trials. Sports Med. 2015; 45:1469-81.

21. Vasan RS, et al. Impact of high-normal blood pressure on the risk of cardiovascular disease. N Engl J Med. 2001;345(18):1291-7.

22. Jin-Ho Y, Wi-Young S. Associations of hypertension status with physical fitness variables in Korean women. Iran J Public Health. 2013;42(7):673.

23. Cornelissen VA, Smart NA. Exercise training for blood pressure: a systematic review and meta-analysis. J Am Heart Assoc. 2013;2(1):e004473.

24. Molmen-Hansen HE, et al. Aerobic interval training reduces blood pressure and improves myocardial function in hypertensive patients. Eur J Prev Cardiol. 2012;19(2):151-60.

25. Pescatello LS, et al. American College of Sports Medicine position stand exercise and hypertension. Med Sci Sports Exerc. 2004;36(3):533-53.

26. Gibala MJ, et al. Physiological adaptations to low-volume, high-intensity interval training in health and disease. J Physiol. 2012;590(5):1077-84. 
27. Gillen JB, Gibala MJ. Is high-intensity interval training a time-efficient exercise strategy to improve health and fitness? Appl Physiol Nutr Metab. 2013;39(3):409-12.

28. Trost SG, Owen N, Bauman AE, Sallis JF, Brown W. Correlates of adults' participation in physical activity: review and update. Med Sci Sports Exerc. 2002:34(12):1996-2001.

29. Macpherson R, et al. Run sprint interval training improves aerobic performance but not maximal cardiac output. Med Sci Sports Exerc. 2011; 43(1):115-22.

30. Willoughby $\mathrm{TN}$, et al. Four weeks of running sprint interval training improves cardiorespiratory fitness in young and middle-aged adults. J Sports Sci. 2016;34(13):1207-14.

31. Boutron I, et al. Extending the CONSORT statement to randomized trials of nonpharmacologic treatment: explanation and elaboration. Ann Intern Med. 2008;148(4):295-309.

32. Bull FC, Maslin TS, Armstrong T. Global physical activity questionnaire (GPAQ): nine country reliability and validity study. J Phys Act Health. 2009; 6(6):790.

33. Ministerio de Salud y Protección Social. Aseguramiento Al Sistema General de Seguridad Social En Salud. https://www.minsalud.gov.co/sites/rid/Lists/ BibliotecaDigital/RIDENP/DOA/RL/cartillas-de-aseguramiento-al-sistemageneral-de-seguridad-social-en-salud.pdf (2014). Accessed 3 September 2015.

34. Saghaei M. Random allocation software for parallel group randomized trials. BMC Med Res Methodol. 2004;4(1):1.

35. Doig GS, Simpson F. Randomization and allocation concealment: a practical guide for researchers. J Crit Care. 2005;20(2):187-91.

36. Garber CE, et al. Quantity and quality of exercise for developing and maintaining cardiorespiratory, musculoskeletal, and neuromotor fitness in apparently healthy adults: guidance for prescribing exercise. Med Sci Sports Exerc. 2011;43(7):1334-59.

37. Lagally KM, Robertson RJ. Construct validity of the OMNI resistance exercise scale. J Strength Cond Res. 2006;20(2):252-6.

38. Hatle $\mathrm{H}$, et al. Effect of 24 sessions of high-intensity aerobic interval training carried out at either high or moderate frequency, a randomized trial. PLoS One. 2014;9(2):e88375.

39. Moholdt $\Pi$, et al. Aerobic interval training versus continuous moderate exercise after coronary artery bypass surgery: a randomized study of cardiovascular effects and quality of life. Am Heart J. 2009;158(6):1031-7.

40. Zwetsloot KA, et al. High-intensity interval training induces a modest systemic inflammatory response in active, young men. J Inflamm Res. 2014;7:9-17.

41. Hasson R, et al. Validity of the Omron HJ-112 pedometer during treadmill walking. Med Sci Sports Exerc. 2009:41(4):805.

42. Alman RE. Cardiorespiratory and health-related physical fitness assessments. In: Swain DP, editor. ACSM's resource manual for quidelines for exercise testing and prescription. Philadelphia, PA: Lippincott Williams \& Wilkins; 2014. p. 335-54

43. Topouchian J, et al. Validation of four automatic devices for selfmeasurement of blood pressure according to the international protocol of the European Society of Hypertension. Vasc Health Risk Manag. 2011;7:709.

44. American College of Sports Medicine. ACSM's resource manual for guidelines for exercise testing and prescription. 9th ed. Philadelphia, PA: Lippincott Williams \& Wilkins; 2013. p. 19-38.

45. Klein $\mathrm{S}$, et al. Waist circumference and cardiometabolic risk: a consensus statement from shaping America's health: Association for Weight Management and Obesity Prevention; NAASO, the Obesity Society; the American Society for Nutrition; and the American Diabetes Association. Obesity. 2007;15(5):1061-7.

46. Darter BJ, Rodriguez KM, Wilken JM. Test-retest reliability and minimum detectable change using the k4b2: oxygen consumption, gait efficiency, and heart rate for healthy adults during submaximal walking. Res Q Exerc Sport. 2013;84(2):223-31.

47. Duffield R, Dawson B, Pinnington HC, Wong P. Accuracy and reliability of a Cosmed K4b² portable gas analysis system. J Sci Med Sport. 2004;7(1):11-22

48. Duffield R, Edge J, Bishop D. Effects of high-intensity interval training on the response during severe exercise. J Sci Med Sport. 2006;9(3):249-55.

49. Pinnington $\mathrm{HC}$, Wong $\mathrm{P}$, Tay J, Green $\mathrm{D}$, Dawson $\mathrm{B}$. The level of accuracy and agreement in measures of $\mathrm{F}_{\mathrm{E}} \mathrm{O}_{2}, \mathrm{~F}_{\mathrm{E}} \mathrm{CO}_{2}$ and $\mathrm{V}_{\mathrm{E}}$ between the Cosmed K4 $\mathrm{b}^{2}$ portable, respiratory gas analysis system and a metabolic cart. J Sci Med Sport. 2001:4(3):324-35.
50. Schrack JA, Simonsick EM, Ferrucci L. Comparison of the Cosmed K4b ${ }^{2}$ portable metabolic system in measuring steady-state walking energy expenditure. PLoS One. 2010;5(2):e9292.

51. Mallén JAC, Piedrafita $E$, Aragonés MT. Criterios de maximalidad en pruebas de esfuerzo. Rev Int Med Cienc Act Fis Deporte. 2009;35:1.

52. Vickers AJ, Altman DG. Analysing controlled trials with baseline and follow up measurements. BMJ. 2001;323(7321):1123-4.

53. Sterne JA, et al. Multiple imputation for missing data in epidemiological and clinical research: potential and pitfalls. BMJ. 2009;338:b2393.

54. Blair SN, et al., Changes in physical fitness and all-cause mortality. A prospective study of healthy and unhealthy men. JAMA. 1995:273(14):1093-8.

55. Kavanagh $T$, et al., Peak oxygen intake and cardiac mortality in women referred for cardiac rehabilitation. J Am Coll Cardiol. 2003:42(12):2139-43.

56. Lee DC, et al., Mortality trends in the general population: the importance of cardiorespiratory fitness. J Psychopharmacol. 2010;24(4 Suppl):27-35.

57. Leitzmann MF, et al., Physical activity recommendations and decreased risk of mortality. Arch Intern Med. 2007;167(22):2453-60.

58. Slattery ML, Jacobs DR Jr., Physical fitness and cardiovascular disease mortality. The US Railroad Study. Am J Epidemiol, 1988;127(3):571-80.

\section{Submit your next manuscript to BioMed Central and we will help you at every step:}

- We accept pre-submission inquiries

- Our selector tool helps you to find the most relevant journal

- We provide round the clock customer support

- Convenient online submission

- Thorough peer review

- Inclusion in PubMed and all major indexing services

- Maximum visibility for your research

Submit your manuscript at www.biomedcentral.com/submit 